\title{
Sharp bounds for eigenvalues of biharmonic operators with complex potentials in low dimensions
}

\author{
Orif O. Ibrogimov, ${ }^{a}$ David Krejčiřík ${ }^{a}$ and Ari Laptev ${ }^{b}$
}

a) Department of Mathematics, Faculty of Nuclear Sciences and Physical Engineering, Czech Technical University, Trojanova 13, 12000 Prague 2, Czech Republic; ibrogori@fjfi.cvut.cz, david.krejcirik@fjfi.cvut.cz.

b) Department of Mathematics, Imperial College London, Huxley Building, 180 Queen's Gate, London SW72AZ, UK; a.laptev@imperial.ac.uk.

28 February 2019

\begin{abstract}
We derive sharp quantitative bounds for eigenvalues of biharmonic operators perturbed by complex-valued potentials in dimensions one, two and three.
\end{abstract}

\section{Introduction}

Spectral theory of self-adjoint operators has exhibited an enormous development since the discovery of quantum mechanics in the beginning of the last century and by now it can be regarded as well understood in many respects. Recent years have brought new motivations for considering non-selfadjoint operators, too, including quantum mechanics again, but this theory is still in its infancy.

A strong impetus for a systematic study of spectral properties of Schrödinger operators with complex-valued potentials goes back to the celebrated result of Davies et al. in 2001 [1] showing that every discrete eigenvalue of the one-dimensional operator $-\Delta \dot{+} V$ lies in the closed disk of the complex plane centred at the origin and with the radius equal to $\frac{1}{4}\|V\|_{L^{1}(\mathbb{R})}^{2}$. This bound is sharp in the sense that there are potentials with eigenvalues lying on the boundary circle. The purpose of this paper is to extend this type of bounds to biharmonic operators

$$
H_{V}:=\Delta^{2} \dot{+} V \quad \text { in } \quad L^{2}\left(\mathbb{R}^{d}\right)
$$

in dimensions $d=1,2,3$. The feature of our results is that our bounds are sharp and quantitative (at least if $d=1,3$ ). Moreover, potentials of minimal regularity are considered and we cover embedded eigenvalues, too.

The pioneering work [1] has been followed by extensive investigations devoted to bounds on the number and magnitudes of eigenvalues of Schrödinger operators with complex-valued potentials. Instead of listing the huge number of articles by various groups on operators not dealt with in this paper, we refer to the recent work [13] containing a rather extensive bibliography. On the other hand, related issues for higher-order partial differential operators seem to be just scarcely considered in the literature, even in the self-adjoint setting. The reader is referred to [20, 23, 9, 10, 7, 17; ;ee also [11, 4, 5, 14, 6] for other spectral questions.

We recall the importance of biharmonic operators in continuum mechanics and in particular linear elasticity theory and the solution of Stokes flows. To the best of our knowledge, the only work where eigenvalue bounds for non-self-adjoint biharmonic operators were considered previously is the recent paper of Enblom [10. We also refer to recent Hulko's paper [17] for bounds on 
the number of discrete eigenvalues of biharmonic operators perturbed by exponentially decaying complex-valued potentials. While the author of [10 proceeds in a rather extensive generality (polyharmonic operators in any dimension), her bounds are not quantitative (in the sense that the involved constants depend on the arguments of the individual eigenvalues under consideration) and embedded eigenvalues are not covered. For these reasons, it is our belief that our results are of interest even if restricted to biharmonic operators in low dimensions only. Moreover, our methodology in principle enables one to obtain analogous results also for higher-order polyharmonic operators.

Before stating our main results, we remark that the operator (1) is introduced in a standard way as an m-accretive operator obtained as a form sum of the bi-Laplacian $H_{0}:=\Delta^{2}$ with domain $H^{2}\left(\mathbb{R}^{d}\right)$ and a relatively form-bounded potential $V: \mathbb{R}^{d} \rightarrow \mathbb{C}$. We refer to Section 2 for more details. More specifically, our standing assumption is that there exist numbers $a \in(0,1)$ and $b \in \mathbb{R}$ such that, for all $\psi \in H^{2}\left(\mathbb{R}^{d}\right)$,

$$
\int_{\mathbb{R}^{d}}|V||\psi|^{2} \leq a \int_{\mathbb{R}^{d}}|\Delta \psi|^{2}+b \int_{\mathbb{R}^{d}}|\psi|^{2} .
$$

In particular, potentials $V \in L^{1}\left(\mathbb{R}^{d}\right)$ are covered by this hypothesis. Furthermore, in the same way, it is also possible to proceed in a greater generality and give a meaning to the distributional Dirac delta potential $\delta$, which is explicitly solvable. Putting $\|\delta\|_{L^{1}\left(\mathbb{R}^{d}\right)}:=1$ by convention, Theorem 1 remains valid in this more general setting.

Our first result is a biharmonic analogue of the celebrated result of [1] for one-dimensional Schrödinger operators.

Theorem 1. Let $d \in\{1,2,3\}$ and assume that $V \in L^{1}\left(\mathbb{R}^{d}\right)$. Then there exists a universal constant $C_{d}>0$ such that

$$
\sigma_{\mathrm{p}}\left(H_{V}\right) \subset\left\{\lambda \in \mathbb{C}:|\lambda| \leq C_{d}\|V\|_{L^{1}\left(\mathbb{R}^{d}\right)}^{4 /(4-d)}\right\}
$$

Moreover, one can take $C_{1}=\frac{1}{4}$ and $C_{3}=\frac{1}{4} \frac{1}{(4 \pi)^{4}}$ for dimensions $d=1$ and $d=3$, respectively.

The theorem is sharp due to the weak-coupling asymptotics

$$
\inf \sigma\left(H_{\beta V}\right)=-c_{d} \beta^{4 /(4-d)}\|V\|_{L^{1}\left(\mathbb{R}^{d}\right)}^{4 /(4-d)}+o\left(\beta^{4 /(4-d)}\right) \quad \text { as } \quad \beta \rightarrow 0^{+}
$$

valid for every sufficiently regular, non-positive, real-valued potential $V$ with $c_{1}:=\frac{1}{4}, c_{2}:=\frac{1}{64}$ and $c_{3}:=\frac{1}{4} \frac{1}{(4 \pi)^{4}}, c f$. [20, Eq. (8)]. We do not know whether one can take $C_{2}=\frac{1}{64}$ in (3) for dimension $d=2$, but it is natural to conjecture so. The result is sharp also in the sense that for $d=1$ (respectively, $d=3$ ) one has $\sigma_{\mathrm{p}}\left(H_{\alpha \delta}\right)=\left\{\frac{1}{4} \alpha^{4 / 3} e^{-i \pi / 3}\right\}$ for every $\alpha \in \mathbb{C}$ such that $\Re \alpha<|\Im \alpha|$ (respectively, $\sigma_{\mathrm{p}}\left(H_{4 \pi \alpha \delta}\right) \supset\left\{-\frac{1}{4} \alpha^{4}\right\}$ if $\left.\Re \alpha<-|\Im \alpha|\right)$.

In the next series of results, we go beyond the $L^{1}$-potentials in the three-dimensional case. First, we employ the Rollnik class of potentials ( $c f$. [22, Ch. I]), which consist of all $V \in L_{\text {loc }}^{1}\left(\mathbb{R}^{3}\right)$ such that

$$
\|V\|_{R\left(\mathbb{R}^{3}\right)}^{2}:=\iint_{\mathbb{R}^{3} \times \mathbb{R}^{3}} \frac{|V(x)||V(y)|}{|x-y|^{2}} d x d y<\infty .
$$

In Section 2, we argue that $V \in R\left(\mathbb{R}^{3}\right)$ is a sufficient condition to guarantee (2), so $H_{V}$ is well defined.

Theorem 2. Let $d=3$ and assume that $V \in R\left(\mathbb{R}^{3}\right)$. Then

$$
\sigma_{\mathrm{p}}\left(H_{V}\right) \subset\left\{\lambda \in \mathbb{C}:|\lambda| \leq \frac{1}{2} \frac{\|V\|_{R\left(\mathbb{R}^{3}\right)}^{2}}{(4 \pi)^{2}}\right\} .
$$

This result has an important consequence for potentials belonging to $L^{3 / 2}\left(\mathbb{R}^{3}\right) \hookrightarrow R\left(\mathbb{R}^{3}\right)$. In fact, using a sharp version of the Hardy-Littlewood-Sobolev inequality ( $c f$. [19, Thm. 4.3]) which quantifies the embedding, the bound (5) immediately implies the following result. 
Corollary 1. Let $d=3$ and assume that $V \in L^{3 / 2}\left(\mathbb{R}^{3}\right)$. Then

$$
\sigma_{\mathrm{p}}\left(H_{V}\right) \subset\left\{\lambda \in \mathbb{C}:|\lambda| \leq \frac{1}{8} \frac{1}{(4 \pi)^{2 / 3}}\|V\|_{L^{3 / 2}\left(\mathbb{R}^{3}\right)}^{2}\right\} .
$$

Finally, we establish the following robust result.

Theorem 3. Let $d=3$ and assume (2). Then

$$
\sigma_{\mathrm{p}}\left(H_{V}\right) \subset\left\{\lambda \in \mathbb{C}:|\lambda| \leq\left(\sup _{\substack{\psi \in H^{1}\left(\mathbb{R}^{3}\right) \\ \psi \neq 0}} \frac{\int_{\mathbb{R}^{3}}|V||\psi|^{2}}{\int_{\mathbb{R}^{3}}|\nabla \psi|^{2}}\right)^{2}\right\} .
$$

This theorem is particularly useful to cover the Hardy-type potentials $x \mapsto|x|^{-2}$, which belong neither to $L^{1}\left(\mathbb{R}^{3}\right)$ nor $R\left(\mathbb{R}^{3}\right)$. For this reason, let us consider the class of potentials, which consist of all $V \in L_{\text {loc }}^{1}\left(\mathbb{R}^{3}\right)$ such that

$$
\|V\|_{H\left(\mathbb{R}^{3}\right)}:=\operatorname{esssup}_{x \in \mathbb{R}^{3}}|x|^{2}|V(x)|<\infty .
$$

Employing the classical Hardy inequality

$$
\int_{\mathbb{R}^{3}}|\nabla \psi|^{2} \geq \frac{1}{4} \int_{\mathbb{R}^{3}} \frac{|\psi(x)|^{2}}{|x|^{2}} d x
$$

valid for every $\psi \in H^{1}\left(\mathbb{R}^{3}\right)$, it is easy to check that $V \in H\left(\mathbb{R}^{3}\right)$ is a sufficient condition to guarantee (2). Furthermore, using (9) in (7), we get the following immediate consequence.

Corollary 2. Let $d=3$ and assume that $V \in H\left(\mathbb{R}^{3}\right)$. Then

$$
\sigma_{\mathrm{p}}\left(H_{V}\right) \subset\left\{\lambda \in \mathbb{C}:|\lambda| \leq 16\|V\|_{H\left(\mathbb{R}^{3}\right)}^{2}\right\} .
$$

In this paper we leave aside dimension $d=4$, which exhibits the same type of difficulties as dimension two for Schrödinger operators. The reason for explicit constants in our one- and three-dimensional theorems is due to the availability of explicit forms of the resolvent kernels of $H_{0}$ in these dimensions. On the other hand, in view of the subcriticality of $H_{0}$ in dimensions $d \geq 5$, a different kind of results is expected in analogy with the case of Schrödinger operators ( $c f$. [18, 15, 13]): the point spectrum of $H_{V}$ should be empty for all potentials which are sufficiently small in a suitable sense. This type of results are also left open in this paper.

The organisation of the paper is as follows. In Section 2 we rigorously introduce the biharmonic operators (11) and determine sufficient conditions on the potential $V$ which guarantee the basic hypothesis (2). In Section 3 we establish the main tool of our approach, namely a sort of the Birman-Schwinger principle covering embedded eigenvalues, too. The proofs of Theorems 10 are given in Section 4. Finally, in Section 5 we introduce explicitly solvable models in terms of Dirac delta potentials and argue about the optimality of Theorem 11 for dimensions $d=1$ and $d=3$.

\section{Definition of biharmonic operators}

First of all, let us comment on the definition of $H_{V}$ given in (1).

Let $H_{0}$ be the the self-adjoint operator in $L^{2}\left(\mathbb{R}^{d}\right)$ associated with the quadratic form

$$
h_{0}[\psi]:=\int_{\mathbb{R}^{d}}|\Delta \psi|^{2}, \quad \mathrm{D}\left(h_{0}\right):=H^{2}\left(\mathbb{R}^{d}\right) .
$$

One has $\mathrm{D}\left(H_{0}\right)=H^{4}\left(\mathbb{R}^{d}\right)$ and $H_{0}=\Delta^{2}$. The spectrum of $H_{0}$ is purely absolutely continuous and coincides with the semi-axis $[0,+\infty)$. If $d \leq 4$, the operator $H_{0}$ is critical in the sense that 
$\inf \sigma\left(H_{0}+V\right)<0$ whenever $V \in C_{0}^{\infty}\left(\mathbb{R}^{d}\right)$ is real-valued, non-positive and non-trivial. On the other hand, if $d \geq 5$, the operator is subcritical in the sense that its spectrum is stable under the non-positive perturbations above; more specifically, $H_{0}$ satisfies a Hardy-type inequality $H_{0} \geq \rho$ in the sense of forms, where $\rho: \mathbb{R}^{d} \rightarrow[0, \infty)$ is non-trivial.

Let $v$ be a quadratic form in $L^{2}\left(\mathbb{R}^{d}\right)$, which is relatively bounded with respect to $h_{0}$ with the relative bound less than one. That is, $\mathrm{D}(v) \supset H^{2}\left(\mathbb{R}^{d}\right)$ and there exist numbers $a \in(0,1)$ and $b \in \mathbb{R}$ such that, for all $\psi \in H^{2}\left(\mathbb{R}^{d}\right)$,

$$
|v[\psi]| \leq a \int_{\mathbb{R}^{d}}|\Delta \psi|^{2}+b \int_{\mathbb{R}^{d}}|\psi|^{2} .
$$

Then the sum $h_{V}:=h_{0}+v$ is a closed sectorial form with $\mathrm{D}\left(h_{V}\right)=H^{2}\left(\mathbb{R}^{d}\right)$, which gives rise to an m-sectorial operator $H_{V}$ in $L^{2}\left(\mathbb{R}^{d}\right)$ via the representation theorem (cf. [18, Thm. VI.2.1]).

For example, if $V \in L_{\mathrm{loc}}^{1}\left(\mathbb{R}^{d}\right)$ is such that

$$
v[\psi]:=\int_{\mathbb{R}^{d}} V|\psi|^{2}, \quad \mathrm{D}(v):=\left\{\psi \in L^{2}\left(\mathbb{R}^{d}\right): \int_{\mathbb{R}^{d}}|V||\psi|^{2}<\infty\right\},
$$

verifies (12) (which coincides with (2) in this case), then we write $H_{V}=H_{0} \dot{+} V$ as in (1) and understand the plus symbol in the sense of forms described above. It is important to keep in mind that $H_{V}$ may differ from the operator sum $H_{0}+V$. Since the adjoint operator satisfies $H_{V}^{*}=H_{\bar{V}}=\mathcal{T} H_{V} \mathcal{T}$, where $\mathcal{T}$ is the complex conjugation operator defined by $\mathcal{T} \psi:=\bar{\psi}, H_{V}$ is a $\mathcal{T}$-self-adjoint operator. Consequently, the residual spectrum of $H_{V}$ is always empty (cf. [8, Sec. III.5]). Furthermore, if $V$ vanishes at infinity in a suitable sense, then (any kind of) the essential spectrum $H_{V}$ coincides with the semi-axis $[0,+\infty)$.

Let us now discuss sufficient conditions which guarantee (12).

By the Sobolev embedding theorem ([3, Thm. 5.4]), every function $\psi \in H^{2}\left(\mathbb{R}^{d}\right)$ is bounded and continuous as long as $d \leq 3$. More specifically ( $c f$. [21, Theorem IX.28]), for any positive $\alpha$ there is $\beta \in \mathbb{R}$ such that, for all $\psi \in H^{2}\left(\mathbb{R}^{d}\right)$,

$$
\|\psi\|_{L^{\infty}\left(\mathbb{R}^{d}\right)} \leq \alpha\|\Delta \psi\|_{L^{2}\left(\mathbb{R}^{d}\right)}+\beta\|\psi\|_{L^{2}\left(\mathbb{R}^{d}\right)} .
$$

Consequently, any potential $V \in L^{1}\left(\mathbb{R}^{d}\right)+L^{\infty}\left(\mathbb{R}^{d}\right)$ satisfies (2) with the relative bound equal to zero (i.e. a can be chosen arbitrarily small). Furthermore, the inequality (13) enables us to give a meaning to the operator $H_{\delta}=H_{0} \dot{+} \delta$, where $\delta$ is the Dirac delta function, by setting $v[\psi]:=|\psi(0)|^{2}$, $\mathrm{D}(v):=H^{2}\left(\mathbb{R}^{d}\right)$.

Rollnik potentials are relatively form-bounded with respect to the Laplacian with the relative bound equal to zero ( $c f$. [21, Theorem X.19]). More specifically, if $V \in R\left(\mathbb{R}^{3}\right)$, then for any positive $\alpha$ there is $\beta \in \mathbb{R}$ such that, for all $\psi \in H^{1}\left(\mathbb{R}^{3}\right)$,

$$
\int_{\mathbb{R}^{3}}|V||\psi|^{2} \leq \alpha\|\nabla \psi\|_{L^{2}\left(\mathbb{R}^{3}\right)}^{2}+\beta\|\psi\|_{L^{2}\left(\mathbb{R}^{3}\right)}^{2} .
$$

Consequently, if additionally $\psi \in H^{2}\left(\mathbb{R}^{3}\right)$, then

$$
\begin{aligned}
\int_{\mathbb{R}^{3}}|V \| \psi|^{2} & \leq \alpha(\psi,-\Delta \psi)_{L^{2}\left(\mathbb{R}^{3}\right)}+\beta\|\psi\|_{L^{2}\left(\mathbb{R}^{3}\right)} \\
& \leq \alpha\|\psi\|_{L^{2}\left(\mathbb{R}^{3}\right)}\|\Delta \psi\|_{L^{2}\left(\mathbb{R}^{3}\right)}+\beta\|\psi\|_{L^{2}\left(\mathbb{R}^{3}\right)}^{2} .
\end{aligned}
$$

From this inequality, it is easy to conclude that any potential $V \in R\left(\mathbb{R}^{3}\right)+L^{\infty}\left(\mathbb{R}^{3}\right)$ satisfies (2) with the relative bound equal to zero.

Finally, if $V \in H\left(\mathbb{R}^{3}\right)$, then the Hardy inequality (9) yields, for all $\psi \in H^{1}\left(\mathbb{R}^{3}\right)$,

$$
\int_{\mathbb{R}^{3}}|V||\psi|^{2} \leq\|V\|_{H\left(\mathbb{R}^{3}\right)} \int_{\mathbb{R}^{3}} \frac{|\psi(x)|^{2}}{|x|^{2}} d x \leq 4\|V\|_{H\left(\mathbb{R}^{3}\right)}\|\nabla \psi\|_{L^{2}\left(\mathbb{R}^{3}\right)}^{2} .
$$

Hence $V$ is form-subordinated with respect to the Laplacian. Proceeding as in (14), we conclude that any potential $V \in H\left(\mathbb{R}^{3}\right)+L^{\infty}\left(\mathbb{R}^{3}\right)$ satisfies (2) with the relative bound equal to zero. 


\section{The Birman-Schwinger principle}

The main role in our proof of Theorems 13 is played by the Birman-Schwinger operator

$$
K_{z}:=|V|^{1 / 2}\left(H_{0}-z\right)^{-1} V_{1 / 2} \quad \text { with } \quad V_{1 / 2}:=|V|^{1 / 2} \operatorname{sgn}(V),
$$

where sgn: $\mathbb{C} \rightarrow \mathbb{C}$ is the complex signum function defined by

$$
\operatorname{sgn}(z):=\left\{\begin{array}{cl}
\frac{z}{|z|} & \text { if } z \neq 0 \\
0 & \text { if } z=0 .
\end{array}\right.
$$

We abuse the notation by using the same symbols for maximal operators of multiplication and their generating functions. The operator $K_{z}$ is well defined (on its natural domain of the composition of three operators) for all $z \in \mathbb{C}$ and $d \geq 1$.

If $z \notin[0,+\infty)$, however, $K_{z}$ is a bounded operator under our hypothesis (2). Indeed, $V_{1 / 2}$ maps $L^{2}\left(\mathbb{R}^{d}\right)$ to $H^{-2}\left(\mathbb{R}^{d}\right)$ by duality, $\left(H_{0}-z\right)^{-1}$ is an isomorphism between $H^{-2}\left(\mathbb{R}^{d}\right)$ and $H^{2}\left(\mathbb{R}^{d}\right)$, and the latter space is mapped by $|V|^{1 / 2}$ back to $L^{2}\left(\mathbb{R}^{d}\right)$. Furthermore, we have a useful formula for the integral kernel of $K_{z}$ :

$$
K_{z}(x, y)=|V|^{1 / 2}(x) \widetilde{G}_{z}(x, y) V_{1 / 2}(y),
$$

where $\widetilde{G}_{z}$ is the Green's function of $H_{0}-z$, i.e. the integral kernel of the resolvent $\left(H_{0}-z\right)^{-1}$. What is more, for dimensions $d=1$ and $d=3$, we have explicit formulae for $\widetilde{G}_{z}$, whereas the latter can be expressed in terms of modified Hankel functions for $d=2$. In fact, using the identity

$$
\left(\Delta^{2}-z\right)^{-1}=\frac{1}{2 k}\left[(-\Delta-k)^{-1}-(-\Delta+k)^{-1}\right]
$$

where $k \in \mathbb{C} \backslash[0, \infty)$ is such that $k^{2}=z$ (throughout the paper we choose the principal branch of the square root), and the well-known formulae for the integral kernels of the resolvent of the Laplacian in these dimensions yield

$$
\widetilde{G}_{z}(x, y)=\frac{1}{2 k}\left[G_{k}(x, y)-G_{-k}(x, y)\right]
$$

where

$$
G_{k}(x, y):= \begin{cases}\frac{e^{-\sqrt{-k}|x-y|}}{2 \sqrt{-k}} & \text { if } \quad d=1, \\ \frac{1}{2 \pi} K_{0}(\sqrt{-k}|x-y|) & \text { if } \quad d=2, \\ \frac{1}{4 \pi} \frac{e^{-\sqrt{-k}|x-y|}}{|x-y|} & \text { if } \quad d=3 .\end{cases}
$$

Here $K_{0}$ is a modified Bessel (also called Macdonald's) function (see [2, Sec. 9] or [16, Sec. 8.4-8.5]).

The following lemma provides an integral criterion for the existence of solutions to the differential eigenvalue equation corresponding to $H_{V}$ and can be considered as a one-sided version of the conventional Birman-Schwinger principle extended to possible eigenvalues embedded in $[0,+\infty)$ as well. Its proof is inspired by the ones of the similar results in [13, 12]. By $\varphi \in L_{0}^{2}\left(\mathbb{R}^{d}\right)$ in the lemma below we mean $\varphi \in L^{2}\left(\mathbb{R}^{d}\right)$ and that $\operatorname{supp} \varphi$ is compact.

Lemma 1. Let $d \in\{1,2,3\}$ and assume (2). If $H_{V} \psi=\lambda \psi$ with some $\lambda \in \mathbb{C}$ and $\psi \in \mathrm{D}\left(H_{V}\right)$, then $\phi:=|V|^{1 / 2} \psi \in L^{2}\left(\mathbb{R}^{d}\right)$ obeys

$$
\forall \varphi \in L_{0}^{2}\left(\mathbb{R}^{d}\right), \quad \lim _{\varepsilon \rightarrow 0^{ \pm}}\left(\varphi, K_{\lambda+i \varepsilon} \phi\right)=-(\varphi, \phi) .
$$


Proof. First, we notice that (2) implies that $\phi \in L^{2}\left(\mathbb{R}^{d}\right)$ whenever $\psi \in \mathrm{D}\left(H_{V}\right) \subset H^{2}\left(\mathbb{R}^{d}\right)$. Let $\varphi \in L_{0}^{2}\left(\mathbb{R}^{d}\right)$ be fixed. Given any $\lambda \in \mathbb{C}$, there is $\varepsilon_{0}>0$ such that $\lambda+i \varepsilon \notin[0,+\infty)$ for all real $\varepsilon$ satisfying $0<|\varepsilon|<\varepsilon_{0}$. We have

$$
\begin{aligned}
\left(\varphi, K_{\lambda+i \varepsilon} \phi\right) & =\iint_{\mathbb{R}^{d} \times \mathbb{R}^{d}} \overline{\varphi(x)}|V|^{1 / 2}(x) G_{\lambda+i \varepsilon}(x, y) V(y) \psi(y) d x d y \\
& =\int_{\mathbb{R}^{d}} \eta_{\varepsilon}(y) V(y) \psi(y) d y
\end{aligned}
$$

where

$$
\eta_{\varepsilon}:=\int_{\mathbb{R}^{d}} \overline{\varphi(x)}|V|^{1 / 2}(x) G_{\lambda+i \varepsilon}(x, \cdot) d x=\left(H_{0}-\lambda-i \varepsilon\right)^{-1}|V|^{1 / 2} \bar{\varphi},
$$

where the second equality holds due to the symmetry $G_{z}(x, y)=G_{z}(y, x)$. In view of (2), $|V|^{1 / 2} \bar{\varphi} \in$ $L^{2}\left(\mathbb{R}^{d}\right)$. Since $\varepsilon \neq 0$ is so small that $\lambda+i \varepsilon \notin \sigma\left(H_{0}\right)$, we have $\eta_{\varepsilon} \in \mathrm{D}\left(H_{0}\right)=H^{4}\left(\mathbb{R}^{d}\right)$. In particular, $\eta_{\varepsilon} \in H^{2}\left(\mathbb{R}^{d}\right)$ and the weak formulation of the eigenvalue equation $H_{V} \psi=\lambda \psi$ yields

$$
\begin{aligned}
\int_{\mathbb{R}^{d}} \eta_{\varepsilon}(y) V(y) \psi(y) d y & =-\left(\Delta \overline{\eta_{\varepsilon}}, \Delta \psi\right)+\lambda\left(\overline{\eta_{\varepsilon}}, \psi\right) \\
& =-\left(\Delta \bar{\psi}, \Delta \eta_{\varepsilon}\right)+\lambda\left(\bar{\psi}, \eta_{\varepsilon}\right) \\
& =-\left(\Delta \bar{\psi}, \Delta \eta_{\varepsilon}\right)+(\lambda+i \varepsilon)\left(\bar{\psi}, \eta_{\varepsilon}\right)-i \varepsilon\left(\bar{\psi}, \eta_{\varepsilon}\right) \\
& =-\left(\bar{\psi},|V|^{1 / 2} \bar{\varphi}\right)-i \varepsilon\left(\bar{\psi}, \eta_{\varepsilon}\right) \\
& =-\left(\varphi,|V|^{1 / 2} \psi\right)-i \varepsilon\left(\overline{\eta_{\varepsilon}}, \psi\right) .
\end{aligned}
$$

Here the last but one equality follows from the weak formulation of the resolvent equation $\left(H_{0}-\lambda-\right.$ $i \varepsilon) \eta_{\varepsilon}=|V|^{1 / 2} \bar{\varphi}$. Consequently, (22) and (23) imply (21) after taking the limit $\varepsilon \rightarrow 0^{ \pm}$, provided that $\varepsilon\left(\bar{\eta}_{\varepsilon}, \psi\right) \rightarrow 0$ as $\varepsilon \rightarrow 0$. To see the latter, we write

$$
\left|\left(\overline{\eta_{\varepsilon}}, \psi\right)\right|=\left|\left(\varphi, M_{\varepsilon} \psi\right)\right| \leq\|\varphi\|\left\|M_{\varepsilon}\right\|\|\psi\|,
$$

where $M_{\varepsilon}:=\chi_{\Omega}|V|^{1 / 2}\left(H_{0}-\lambda-i \varepsilon\right)^{-1}$ with $\Omega:=\operatorname{supp} \varphi$, and it remains to show that $\varepsilon\left\|M_{\varepsilon}\right\|$ tends to zero as $\varepsilon \rightarrow 0$. Following [22, Thm. III.6], we use the resolvent kernels (19) with (20) and the estimate $\left\|M_{\varepsilon}\right\| \leq\left\|M_{\varepsilon}\right\|_{\mathrm{HS}}$.

For $d=1$, we have

$$
\begin{aligned}
\left\|M_{\varepsilon}\right\|_{\mathrm{HS}}^{2} & =\frac{1}{4|k|^{2}} \iint_{\Omega \times \mathbb{R}}|V(x)|\left|G_{k}(x, y)-G_{-k}(x, y)\right|^{2} d x d y \\
& \leq \frac{1}{2|k|^{2}} \iint_{\Omega \times \mathbb{R}}|V(x)|\left[\frac{e^{-2 \Re \sqrt{-k}|x-y|}}{4|k|}+\frac{e^{-2 \Re \sqrt{k}|x-y|}}{4|k|}\right] d x d y \\
& =\frac{1}{8|k|^{3}}\left[\frac{1}{\Re \sqrt{-k}}+\frac{1}{\Re \sqrt{k}}\right] \int_{\Omega}|V(x)| d x,
\end{aligned}
$$

where the last integral is bounded because $V \in L_{\text {loc }}^{1}(\mathbb{R})$ as a consequence of (2). Elementary calculations show that $|k|^{3} \Re \sqrt{ \pm k}$ can not decay faster than $|\varepsilon|^{7 / 4}$ as $\varepsilon \rightarrow 0$. Hence, we have $\left\|M_{\varepsilon}\right\|=\mathcal{O}\left(|\varepsilon|^{-7 / 8}\right)$ as $\varepsilon \rightarrow 0$, which concludes the proof of the lemma for $d=1$.

For $d=3$, we have analogous estimates

$$
\begin{aligned}
\left\|M_{\varepsilon}\right\|_{\mathrm{HS}}^{2} & \leq \frac{1}{32 \pi^{2}|k|^{2}} \iint_{\Omega \times \mathbb{R}^{3}}|V(x)|\left[\frac{e^{-2 \Re \sqrt{-k}|x-y|}}{|x-y|^{2}}+\frac{e^{-2 \Re \sqrt{k}|x-y|}}{|x-y|^{2}}\right] d x d y \\
& =\frac{1}{16 \pi|k|^{2}}\left[\frac{1}{\Re \sqrt{-k}}+\frac{1}{\Re \sqrt{k}}\right] \int_{\Omega}|V(x)| d x .
\end{aligned}
$$

Since $|k|^{2} \Re \sqrt{ \pm k}$ can not decay faster than $|\varepsilon|^{5 / 4}$ as $\varepsilon \rightarrow 0$, we have $\left\|M_{\varepsilon}\right\|=\mathcal{O}\left(|\varepsilon|^{-5 / 8}\right)$ as $\varepsilon \rightarrow 0$. This concludes the proof of the lemma for $d=3$. 
For $d=2$, we need to do rather involved estimates. Using the integral representation of the Macdonald's function ( $c f$. [16, $\S 8.432]$ ), we can write Green's function for the biharmonic operator as

$$
\widetilde{G}_{z}(x, y)=\frac{1}{4 \pi k} \int_{1}^{\infty} \frac{e^{-\sqrt{-k}|x-y| t}-e^{-\sqrt{k}|x-y| t}}{\sqrt{t^{2}-1}} d t .
$$

Next, we observe that, for $t>1$,

$$
\int_{0}^{\infty}\left|e^{-\sqrt{-k} t s}+e^{-\sqrt{k} t s}\right|^{2} d s \leq 2 \int_{0}^{\infty}\left(e^{-2 \Re \sqrt{-k} t s}+e^{-2 \Re \sqrt{k} t s}\right) d s=\frac{1}{t}\left[\frac{1}{\Re \sqrt{k}}+\frac{1}{\Re \sqrt{-k}}\right] .
$$

In view of this estimate, the Minkowski inequality yields

$$
\begin{aligned}
\left\|M_{\varepsilon}\right\|_{\mathrm{HS}}^{2} & \leq \frac{1}{8 \pi^{2}|k|^{2}}\left(\int_{\Omega}|V(x)| d x\right)\left(\int_{1}^{\infty}\left(\int_{0}^{\infty}\left|e^{-\sqrt{-k} t s}+e^{-\sqrt{k} t s}\right|^{2} d s\right)^{1 / 2} \frac{d t}{\sqrt{t^{2}-1}}\right)^{2} \\
& =\frac{1}{8 \pi^{2}|k|^{2}}\left[\frac{1}{\Re \sqrt{-k}}+\frac{1}{\Re \sqrt{k}}\right]\left(\int_{\Omega}|V(x)| d x\right)\left(\int_{1}^{\infty} \frac{1}{\sqrt{t} \sqrt{t^{2}-1}} d t\right)^{2} .
\end{aligned}
$$

We already know that $|k|^{2} \Re \sqrt{ \pm k}$ can not decay faster than $|\varepsilon|^{5 / 4}$ as $\varepsilon \rightarrow 0$. Hence, again we have $\left\|M_{\varepsilon}\right\|=\mathcal{O}\left(|\varepsilon|^{-5 / 8}\right)$ as $\varepsilon \rightarrow 0$, which concludes the proof of the lemma for $d=2$.

The preceding lemma can be viewed as a precise statement of one side of the Birman-Schwinger principle under the minimal regularity assumption (2) on the potential. It roughly says that if $\lambda$ is an eigenvalue of $H_{V}$, then -1 is an eigenvalue of an integral equation related to $K_{\lambda}$. If $\lambda \notin \sigma\left(H_{0}\right)$ the converse implication also holds, but it is not generally true if $\lambda \in \sigma\left(H_{0}\right)$ (cf. [22, Sec. III.2]) and it is not needed for the purpose of this paper. In fact, we exclusively use the following corollary of Lemma 1

Corollary 3. Let $d \in\{1,2,3\}$ and assume (2). Let $\lambda \in \mathbb{C}$ be arbitrary. If either $\lambda \in \rho\left(H_{0}\right)$ and $\left\|K_{\lambda}\right\|<1$, or $\lambda \in \sigma\left(H_{0}\right)$ and $\liminf _{\varepsilon \rightarrow 0^{ \pm}}\left\|K_{\lambda+i \varepsilon}\right\|<1$, then $\lambda \notin \sigma_{\mathrm{p}}\left(H_{V}\right)$.

Proof. Let $\lambda \in \sigma_{\mathrm{p}}\left(H_{V}\right)$, let $\psi$ be a corresponding eigenfunction and set $\phi:=|V|^{1 / 2} \psi$. If it were the case that $\phi=0$, then the definition of $H_{V}$ would yield

$$
\left(\varphi,\left(H_{0}-\lambda\right) \psi\right)=\left(\varphi,\left(H_{V}-\lambda\right) \psi\right)-\int_{\mathbb{R}^{d}} \bar{\varphi} V \psi=0
$$

for all $\varphi \in H^{2}\left(\mathbb{R}^{d}\right)$, and consequently, $H_{0} \psi=\lambda \psi$. Unless $\psi=0$, this would mean that $\lambda$ is an eigenvalue of $H_{0}$, which is impossible. Hence, we conclude that $\phi \neq 0$.

Next, if $\lambda \in \rho\left(H_{0}\right)$, then Lemma 1 with $\varepsilon=0$ implies

$$
\|\phi\|^{2}\left\|K_{\lambda}\right\| \geq\left|\left(\phi, K_{\lambda} \phi\right)\right|=\|\phi\|^{2}
$$

and thus $\left\|K_{\lambda}\right\| \geq 1$.

If $\lambda \in \sigma\left(H_{0}\right)$, we set $\phi_{n}:=\xi_{n} \phi$ for every positive $n$, where $\xi_{n}(x):=\xi(x / n)$ and $\xi \in C_{0}^{\infty}\left(\mathbb{R}^{d}\right)$ is a usual cut-off function satisfying $\xi(x)=1$ for $|x| \leq 1$ and $\xi(x)=0$ for $|x| \geq 2$. As in (29), we have

$$
\left\|\phi_{n}\right\|\|\phi\|\left\|K_{\lambda+i \varepsilon}\right\| \geq\left|\left(\phi_{n}, K_{\lambda+i \varepsilon} \phi\right)\right| \text {. }
$$

In view of $\phi_{n} \in L_{0}^{2}\left(\mathbb{R}^{d}\right)$, we can invoke Lemma 1 and take the limit $\varepsilon \rightarrow 0^{ \pm}$to conclude

$$
\left\|\phi_{n}\right\|\|\phi\| \liminf _{\varepsilon \rightarrow 0^{ \pm}}\left\|K_{\lambda+i \varepsilon}\right\| \geq\left|\left(\phi_{n}, \phi\right)\right|
$$

The desired claim now follows by taking the limit $n \rightarrow \infty$. 


\section{Proofs}

First, we establish two elementary inequalities which will be crucial in obtaining sharp eigenvalue bounds.

Lemma 2. For all non-negative real numbers $p$ and $q$, the following inequality holds

$$
\frac{1}{2}\left(e^{q-p}+e^{p-q}\right) \leq e^{p+q}-\sin (p+q) .
$$

Proof. Let us rewrite (32) as

$$
e^{-2 p}+e^{-2 q}+2 e^{-(p+q)} \sin (p+q) \leq 2 .
$$

Since (33) is symmetric with respect to $p$ and $q$, there is no loss of generality in assuming that $0 \leq p \leq q$. Let us fix $p=p_{0} \geq 0$ and analyse the smooth function

$$
\Phi(q):=e^{-2 p_{0}}+e^{-2 q}+2 e^{-\left(p_{0}+q\right)} \sin \left(p_{0}+q\right)
$$

on the interval $\left[p_{0}, \infty\right)$. It is easy to check that every possible critical point $q_{0}$ of $\Phi$ must satisfy the equation

$$
e^{q_{0}-p_{0}}=\cos \left(p_{0}+q_{0}\right)-\sin \left(p_{0}+q_{0}\right) .
$$

In view of this observation, some elementary calculations yield that

$$
\Phi\left(q_{0}\right)=2 e^{-2 p_{0}}\left(1-\sin ^{2}\left(p_{0}+q_{0}\right)\right) \leq 2 .
$$

On the other hand, we have

$$
\Phi\left(p_{0}\right)=2 e^{-2 p_{0}}\left(1+\sin \left(2 p_{0}\right)\right) \leq 2, \quad \lim _{q \uparrow \uparrow+\infty} \Phi(q)=e^{-2 p_{0}} \leq 1,
$$

the first estimate being the consequence of the elementary inequality $\sin (t) \leq t \leq e^{t}-1$ for $t \geq 0$. Hence, we conclude from (36) and (37) that

$$
\max _{q \geq p_{0}} \Phi(q) \leq 2,
$$

which proves the claim in (33).

Lemma 3. For all non-negative real numbers $p$ and $q$, the following inequality holds

$$
\frac{1}{2}\left(e^{q-p}+e^{p-q}\right) \leq\left(p^{2}+q^{2}\right) e^{p+q}+\cos (p+q) .
$$

Proof. Let us rewrite (39) as

$$
e^{-2 p}+e^{-2 q}-2 e^{-(p+q)} \cos (p+q) \leq 2\left(p^{2}+q^{2}\right) .
$$

Since (40) is symmetric with respect to $p$ and $q$, there is no loss of generality in assuming that $0 \leq p \leq q$. Let us fix $p=p_{0} \geq 0$ and analyse the smooth function

$$
\Phi(q):=e^{-2 p_{0}}+e^{-2 q}-2 e^{-\left(p_{0}+q\right)} \cos \left(p_{0}+q\right)-2\left(p_{0}^{2}+q^{2}\right)
$$

on the interval $\left[p_{0}, \infty\right)$. We have

$$
\Phi^{\prime}(q)=-2 e^{-2 q}+2 e^{-\left(p_{0}+q\right)} \cos \left(p_{0}+q\right)+2 e^{-\left(p_{0}+q\right)} \sin \left(p_{0}+q\right)-4 q .
$$

On the other hand, for all $s, t \in \mathbb{R}$, we have the well-known inequalities $e^{t} \geq \cos (t)+\sin (t)$ and $e^{s} \geq 1+s$. Applying these inequalities with $t=p+q$ and $s=-2 q$, we obtain

$$
\Phi^{\prime}(q) \leq-2 e^{-2 q}+2-4 q \leq 0 .
$$


Therefore, $\Phi$ is non-increasing on $[0, \infty)$ and thus we have

$$
\Phi(q) \leq \Phi\left(p_{0}\right)=2 e^{-2 p_{0}}+2 e^{-2 p_{0}} \cos \left(2 p_{0}\right)-4 p_{0}^{2} \leq 0,
$$

for all $q \geq p_{0}$, where for the last step one needs to recall the inequality

$$
1-\cos (t) \leq \frac{1}{2} t^{2} \leq \frac{1}{2} t^{2} e^{t}, \quad t \geq 0 .
$$

Hence, for each fixed $p \geq 0$, we have $\Phi(q) \leq 0$ for all $q \geq p$. This proves the desired claim.

Remark 1. We note that the expressions on the left-hand-sides of (33) and (40) are non-negative for all $p, q \geq 0$. In the same way as in Lemma 2 one can show that

$$
\frac{1}{2}\left(e^{q-p}+e^{p-q}\right) \leq e^{p+q}+\cos (p+q)
$$

holds for all $p, q \geq 0$.

Now we are in a position to establish our theorems.

\subsection{Proof of Theorem 1}

We start with the case $\lambda \in \mathbb{C} \backslash[0, \infty)$ and denote by $k$ the principal square root of $\lambda$ as before. Throughout the proof we assume the parameter $k$ to be fixed.

First, for each $d \in\{1,2,3\}$, we justify the existence of a universal constant $c_{d}>0$ such that the corresponding Green's function of the biharmonic operators obeys the following pointwise estimate

$$
\left|\widetilde{G}_{\lambda}(x, y)\right| \leq \frac{c_{d}}{|k|^{2-d / 2}} .
$$

For the case $d=1$, elementary calculations show that the inequality

$$
\left|\sqrt{k} e^{-\sqrt{-k}|x-y|}-\sqrt{-k} e^{-\sqrt{k}|x-y|}\right| \leq \sqrt{2} \sqrt{|k|}
$$

is equivalent to (32) with $p=\Re \sqrt{k}|x-y| \geq 0$ and $q=\Im \sqrt{k}|x-y| \geq 0$ if $\arg (\lambda) \in(0, \pi]$ or with $p=\Re \sqrt{-k}|x-y| \geq 0$ and $q=\Im \sqrt{-k}|x-y| \geq 0$ if $\arg (\lambda) \in(-\pi, 0)$. Hence, we have (47) with the constant $c_{1}:=\frac{1}{2 \sqrt{2}}$, i.e.

$$
\left|\widetilde{G}_{\lambda}(x, y)\right| \leq \frac{1}{2 \sqrt{2}|k|^{3 / 2}} .
$$

For the case $d=3$, elementary calculations show that the inequality

$$
\left|e^{-\sqrt{-k}|x-y|}-e^{-\sqrt{k}|x-y|}\right| \leq \sqrt{2} \sqrt{|k|}|x-y|
$$

is equivalent to (39) with $p=\Re \sqrt{k}|x-y| \geq 0$ and $q=\Im \sqrt{k}|x-y| \geq 0$ if $\arg (\lambda) \in(0, \pi]$ or with $p=\Re \sqrt{-k}|x-y| \geq 0$ and $q=\Im \sqrt{-k}|x-y| \geq 0$ if $\arg (\lambda) \in(-\pi, 0)$. That is why we have (477) with the constant $c_{3}:=\frac{1}{4 \sqrt{2} \pi}$, i.e.

$$
\left|\widetilde{G}_{\lambda}(x, y)\right| \leq \frac{1}{4 \sqrt{2} \pi \sqrt{|k|}}
$$

For the case $d=2$, on the account of the asymptotic expansion of the Macdonald's function $K_{0}(\zeta)$ for small $\zeta$, we can write $(c f$. [16, $\left.\S 8.447]\right)$

$$
G_{k}(x, y)=-\frac{1}{2 \pi} \ln \sqrt{-k}-\frac{1}{2 \pi}\left(\gamma+\ln \frac{|x-y|}{2}\right)+\mathrm{o}(|x-y|), \quad|x-y| \rightarrow 0^{+},
$$


where $\gamma$ is the Euler constant. Hence, it follows that

$$
\begin{aligned}
G_{k}(x, y)-G_{-k}(x, y) & =\frac{1}{2 \pi}(\ln \sqrt{k}-\ln \sqrt{-k})+\mathrm{o}(|x-y|) \\
& =-\frac{i}{4}+o(|x-y|), \quad|x-y| \rightarrow 0^{+},
\end{aligned}
$$

and consequently, recalling (19), we get

$$
\widetilde{G}_{\lambda}(x, y)=\frac{i}{8 \pi k} \operatorname{Arg}(k)+o(|x-y|), \quad|x-y| \rightarrow 0^{+} .
$$

On the other hand, in view of the well-known asymptotic expansion of the Macdonald's function $K_{0}(\zeta)$ for large $\zeta$, we have

$$
G_{k}(x, y)=\sqrt{\frac{\pi}{2 \sqrt{-k}|x-y|}} e^{-\sqrt{-k}|x-y|}(1+\mathcal{O}(1))=\mathcal{O}\left(\frac{1}{\sqrt[4]{|x-y|}}\right), \quad|x-y| \rightarrow+\infty,
$$

and consequently,

$$
\widetilde{G}_{\lambda}(x, y)=\mathcal{O}(1), \quad|x-y| \rightarrow+\infty .
$$

Since these asymptotic expansions are uniform in $k$ and the Macdonald's function is entire on the right half-plane, we conclude the existence of a universal constant $c_{2}>0$ satisfying (47).

In view of (47), now we can estimate the norm of the Birman-Schwinger operator as follows

$$
\left\|K_{\lambda}\right\|^{2} \leq\left\|K_{\lambda}\right\|_{\mathrm{HS}}^{2}=\iint_{\mathbb{R}^{d} \times \mathbb{R}^{d}}|V(x)|\left|\widetilde{G}_{\lambda}(x, y)\right|^{2}|V(y)| d x d y \leq \frac{c_{d}^{2}}{|k|^{4-d}}\|V\|_{L^{1}\left(\mathbb{R}^{d}\right)}^{2}
$$

and thus

$$
\left\|K_{\lambda}\right\| \leq \frac{c_{d}\|V\|_{L^{1}\left(\mathbb{R}^{d}\right)}}{|\lambda|^{1-d / 4}}
$$

If $\lambda \in(0, \infty)$, then the same analysis applied for $\lambda+i \varepsilon$ with $\varepsilon>0$ (instead of $\lambda$ ) yields

$$
\liminf _{\varepsilon \rightarrow 0^{ \pm}}\left\|K_{\lambda+i \varepsilon}\right\| \leq \liminf _{\varepsilon \rightarrow 0^{ \pm}} \frac{c_{d}\|V\|_{L^{1}\left(\mathbb{R}^{d}\right)}}{|\lambda+i \varepsilon|^{1-d / 4}}=\frac{c_{d}\|V\|_{L^{1}\left(\mathbb{R}^{d}\right)}}{|\lambda|^{1-d / 4}} .
$$

Hence, Corollary 3 implies that $\lambda \in \mathbb{C} \backslash\{0\}$ cannot be an eigenvalue for $H_{V}$ unless it holds that

$$
c_{d}\|V\|_{L^{1}\left(\mathbb{R}^{d}\right)} \geq|\lambda|^{1-d / 4} .
$$

This completes the proof since the origin $\lambda=0$ obviously belongs to the right-hand side of (3).

\subsection{Proof of Theorem 2}

First, assume that $\lambda \in \mathbb{C} \backslash[0, \infty)$ and let $k$ be the principal square root of $\lambda$. Elementary calculations show that the estimate

$$
\left|e^{-\sqrt{-k}|x-y|}-e^{-\sqrt{k}|x-y|}\right| \leq \sqrt{2}
$$

is equivalent to (46)) with $p=\Re \sqrt{k}|x-y| \geq 0, q=\Im \sqrt{k}|x-y| \geq 0$ if $\arg (\lambda) \in(0, \pi]$, and with $p=\Re \sqrt{-k}|x-y| \geq 0, q=\Im \sqrt{-k}|x-y| \geq 0$ if $\arg (\lambda) \in(-\pi, 0)$. Hence, the Green's function can be estimated as

$$
\left|\widetilde{G}_{\lambda}(x, y)\right| \leq \frac{1}{4 \sqrt{2} \pi|k||x-y|}
$$

for all distinct $x, y \in \mathbb{R}^{3}$. Using this, we can estimate the norm of the Birman-Schwinger operator in terms of the Rollnik norm of $V$ as follows

$$
\left\|K_{\lambda}\right\|^{2} \leq\left\|K_{\lambda}\right\|_{\mathrm{HS}}^{2}=\iint_{\mathbb{R}^{3} \times \mathbb{R}^{3}}|V(x)|\left|\widetilde{G}_{\lambda}(x, y)\right|^{2}|V(y)| d x d y \leq \frac{1}{32 \pi^{2}|k|^{2}}\|V\|_{R}^{2} .
$$


Hence, we have

$$
\left\|K_{\lambda}\right\| \leq \frac{1}{4 \pi \sqrt{|\lambda|}} \frac{\|V\|_{R}}{\sqrt{2}} .
$$

If $\lambda \in(0, \infty)$, then the same analysis applied for $\lambda+i \varepsilon$ with $\varepsilon>0$ (instead of $\lambda$ ) yields that

$$
\liminf _{\varepsilon \rightarrow 0^{ \pm}}\left\|K_{\lambda+i \varepsilon}\right\| \leq \liminf _{\varepsilon \rightarrow 0^{ \pm}} \frac{1}{4 \pi \sqrt{|\lambda+i \varepsilon|}} \frac{\|V\|_{R}}{\sqrt{2}}=\frac{1}{4 \pi \sqrt{|\lambda|}} \frac{\|V\|_{R}}{\sqrt{2}} .
$$

Hence, Corollary 3 implies that $\lambda \backslash\{0\}$ cannot be an eigenvalue for $H_{V}$ if

$$
\frac{1}{4 \pi} \frac{\|V\|_{R}}{\sqrt{2}}<\sqrt{|\lambda|}
$$

This completes the proof since $\lambda=0$ obviously belongs to the right-hand-side of (5).

\subsection{Proof of Theorem 3}

First, assume that $\lambda \in \mathbb{C} \backslash[0, \infty)$ and let $k$ be the principal square root of $\lambda$. By the triangle inequality, we have

$$
\left\|K_{\lambda}\right\| \leq \frac{1}{2|k|}\left[\left\||V|^{1 / 2}(-\Delta-k)^{-1} V_{1 / 2}\right\|+\left\||V|^{1 / 2}(-\Delta+k)^{-1} V_{1 / 2}\right\|\right] .
$$

where the integral kernel reads now $(c f .(20)$ )

$$
(-\Delta-k)^{-1}(x, y)=\frac{e^{-\sqrt{-k}|x-y|}}{4 \pi|x-y|} .
$$

Using the pointwise estimate

$$
\left|(-\Delta-k)^{-1}(x, y)\right| \leq(-\Delta)^{-1}(x, y)
$$

valid for all $x, y \in \mathbb{R}$ with $x \neq y$ and $k \in \mathbb{C}$, we estimate as in [13, proof of Lem. 1]

$$
\left\||V|^{1 / 2}(-\Delta \pm k)^{-1} V_{1 / 2}\right\| \leq\left\||V|^{1 / 2}(-\Delta)^{-1}|V|^{1 / 2}\right\| \leq\left\||V|^{1 / 2}(-\Delta)^{-1 / 2}\right\|\left\|(-\Delta)^{-1 / 2}|V|^{1 / 2}\right\| .
$$

Finally, noticing that

$$
\left\|(-\Delta)^{-1 / 2}|V|^{1 / 2}\right\|=\left\||V|^{1 / 2}(-\Delta)^{-1 / 2}\right\|=\left(\sup _{\substack{\psi \in H^{1}\left(\mathbb{R}^{3}\right) \\ \psi \neq 0}} \frac{\int_{\mathbb{R}^{3}}|V \| \psi|^{2}}{\int_{\mathbb{R}^{3}}|\nabla \psi|^{2}}\right)^{1 / 2}
$$

we arrive at the estimate

$$
\left\|K_{\lambda}\right\| \leq \frac{1}{\sqrt{|\lambda|}}\left(\sup _{\substack{\psi \in H^{1}\left(\mathbb{R}^{3}\right) \\ \psi \neq 0}} \frac{\int_{\mathbb{R}^{3}}|V||\psi|^{2}}{\int_{\mathbb{R}^{3}}|\nabla \psi|^{2}}\right)
$$

We notice as before that the origin $\lambda=0$ trivially satisfies the estimate in (7). If $\lambda \in(0, \infty)$, then by repeating the analysis for $\lambda+i \varepsilon$ with $\varepsilon>0$ (instead of $\lambda$ ) we see that the right-hand side of (72) dominates $\liminf \operatorname{in}_{\varepsilon \rightarrow 0^{ \pm}}\left\|K_{\lambda+i \varepsilon}\right\|$. The claimed inclusion thus immediately follows from Corollary 3 . 


\section{Optimality of the bounds}

In this section we discuss two concrete examples which demonstrate the optimality of the constants corresponding to the eigenvalue bounds for dimensions $d=1$ and $d=3$ in Theorem [1. Both examples are given in terms of distributional Dirac delta potentials, but an approximation by regular potentials is also mentioned. We do not consider the delta potential in two dimensions because the constant $C_{2}$ of Theorem 1 is not explicit (nonetheless, preliminary computations confirm that the weak-coupling constant $c_{2}=\frac{1}{64}$ is achieved by the Dirac delta potential also for $d=2)$.

\subsection{The delta potential in one dimension}

As described in Section 2, the operator $H_{\alpha \delta}=H_{0} \dot{+} \alpha \delta$ in $L^{2}(\mathbb{R})$ with $\alpha \in \mathbb{C}$ should be understood as the operator associated with the quadratic form

$$
h_{\alpha \delta}[\psi]:=\int_{\mathbb{R}}\left|\psi^{\prime \prime}\right|^{2}+\alpha|\psi(0)|^{2}, \quad \mathrm{D}\left(h_{\alpha \delta}\right):=H^{2}(\mathbb{R}) .
$$

One has

$$
\begin{aligned}
H_{\alpha \delta} \psi(x) & =\psi^{\prime \prime \prime \prime}(x), \quad x \in \mathbb{R} \backslash\{0\}, \\
\mathrm{D}\left(H_{\alpha \delta}\right) & =\left\{\psi \in H^{4}(\mathbb{R} \backslash\{0\}) \cap H^{3}(\mathbb{R}): \psi^{\prime \prime \prime}\left(0^{+}\right)-\psi^{\prime \prime \prime}\left(0^{-}\right)=-\alpha \psi(0)\right\} .
\end{aligned}
$$

Note that the function values at 0 are well defined due to the Sobolev embedding $H^{4}(\mathbb{R}) \hookrightarrow C^{3}(\mathbb{R})$. Since the perturbation is a point interaction, it follows that $\sigma_{\mathrm{ess}}\left(H_{\alpha \delta}\right)=[0,+\infty)$.

Let us look for eigenvalues of $H_{\alpha \delta}$, i.e. we consider $H_{\alpha \delta} \psi=k^{4} \psi$ with the convention

$$
\Re k>0 \quad \text { and } \quad \Im k>0 \text {. }
$$

The general solution of the differential equation $\psi^{\prime \prime \prime \prime}=k^{4} \psi$ in any open real interval reads

$$
\psi(x)=C_{1} e^{k x}+C_{2} e^{-k x}+C_{3} e^{i k x}+C_{4} e^{-i k x},
$$

where $C_{1}, C_{2}, C_{3}, C_{4}$ are arbitrary complex numbers. Considering these general solution in the separate intervals $(-\infty, 0)$ and $(0,+\infty)$, and imposing the integrability requirements $\left(C_{1}, C_{4}=0\right.$ if $x>0$ and $C_{2}, C_{3}=0$ if $x<0$ ) together with the interface conditions at zero due to (74) (continuity up to the second derivative and the jump condition for the third derivative), we arrive at the condition

$$
k^{3}=\frac{1}{2^{3 / 2}} \alpha e^{-i \pi / 4} .
$$

In order to make this equation compatible with (175), we see that the point spectrum of $H_{\alpha \delta}$ is not empty if, and only if, $\arg \alpha \in(\pi / 4,7 \pi / 4)$, or equivalently, $\Re \alpha<|\Im \alpha|$. If this condition is satisfied, $H_{\alpha \delta}$ possesses one eigenvalue corresponding to the right-hand side put to the power $4 / 3$. Summing up,

$$
\sigma_{\mathrm{p}}\left(H_{\alpha \delta}\right)=\left\{\begin{array}{lll}
\left\{\frac{1}{4} \alpha^{4 / 3} e^{-i \pi / 3}\right\} & \text { if } & \Re \alpha<|\Im \alpha|, \\
\varnothing & \text { if } \quad \Re \alpha \geq|\Im \alpha| .
\end{array}\right.
$$

Notice that the arguments of $\alpha$ for which the point spectrum is empty correspond to real points intersecting the half-axis $[0,+\infty)$. So any eigenvalue of $H_{\alpha \delta}$, if it exists, is necessarily discrete.

In particular, choosing $\alpha:=e^{i \theta}$ and varying $\theta \in(\pi / 4,7 \pi / 4)$, any boundary point of the closed disk $\left\{\lambda \in \mathbb{C}:|\lambda| \leq \frac{1}{4}\right\}$ except for the point $\lambda=\frac{1}{4}$ will be an eigenvalue of $H_{\alpha \delta}$. This proves the optimality of Theorem 1 for $d=1$ because $\|\alpha \delta\|_{L^{1}(\mathbb{R})}=|\alpha|=1$. 
Remark 2. One can also justify the optimality of the constant in the eigenvalue bound corresponding to the inclusion (3) for $d=1$ in the limit $\varepsilon \rightarrow 0^{+}$by considering the following family of regular but singularly scaled potentials

$$
\delta_{\varepsilon}(x):= \begin{cases}\frac{1}{\varepsilon} & \text { if }|x|<\varepsilon / 2 \\ 0 & \text { otherwise }\end{cases}
$$

Of course $\delta_{\varepsilon} \rightarrow \delta$ in the sense of distributions as $\varepsilon \rightarrow 0^{+}$. What is more, the point spectrum of $H_{\alpha \delta_{\varepsilon}}$ converges to the point spectrum of $H_{\alpha \delta}$ as $\varepsilon \rightarrow 0^{+}$. This can be checked by explicitly solving the differential equations of the eigenvalue problem in the separate intervals where $\delta_{\varepsilon}$ is constant and matching the solutions at the boundary points.

\subsection{The delta potential in three dimensions}

In analogy with the one-dimensional situation above, we consider the operator $H_{4 \pi \alpha \delta}=H_{0} \dot{+} 4 \pi \alpha \delta$ in $L^{2}\left(\mathbb{R}^{3}\right)$ with $\alpha \in \mathbb{C}$ associated with the quadratic form

$$
h_{4 \pi \alpha \delta}[\psi]:=\int_{\mathbb{R}^{3}}|\Delta \psi|^{2}+4 \pi \alpha|\psi(0)|^{2}, \quad \mathrm{D}\left(h_{4 \pi \alpha \delta}\right):=H^{2}\left(\mathbb{R}^{3}\right) .
$$

Again, $\sigma_{\text {ess }}\left(H_{4 \pi \alpha \delta}\right)=[0,+\infty)$.

Let us look for eigenvalues of $H_{4 \pi \alpha \delta}$ corresponding to radially symmetric eigenfunctions. That is, we consider the equation $H_{4 \pi \alpha \delta} \psi=k^{4} \psi$ with the same convention (75) as above and look for special solutions of the form $\psi(x)=g(|x|)$ with $g \in H^{2}\left((0, \infty), r^{2} d r\right)$. These eigenvalues are determined by eigenvalues of the one dimensional operator $T_{\alpha}$ in $L^{2}\left((0, \infty), r^{2} d r\right)$ associated with the quadratic form

$$
t_{\alpha}[g]:=\int_{0}^{\infty}\left|r^{-1}\left[r g^{\prime}(r)\right]^{\prime}\right|^{2} r^{2} d r+\alpha|g(0)|^{2}, \quad \mathrm{D}\left(t_{\alpha}\right):=H^{2}\left((0, \infty), r^{2} d r\right) .
$$

Using the unitary transform $U: L^{2}\left((0, \infty), r^{2} d r\right) \rightarrow L^{2}((0, \infty), d r)$ which acts as $(U g)(r):=r g(r)$, the operator $T_{\alpha}$ is unitarily equivalent to the operator $\widetilde{T}_{\alpha}:=U T_{\alpha} U^{-1}$ in $L^{2}((0, \infty))$ associated with the quadratic form

$$
\widetilde{t}_{\alpha}[f]:=\int_{0}^{\infty}\left|f^{\prime \prime}(r)\right|^{2} d r+\alpha\left|f^{\prime}(0)\right|^{2}, \quad \mathrm{D}\left(\widetilde{t}_{\alpha}\right):=H^{2}((0, \infty)) \cap H_{0}^{1}((0, \infty)) .
$$

One has

$$
\begin{aligned}
\widetilde{T}_{\alpha} f(r) & =f^{\prime \prime \prime \prime}(r), \quad x \in(0, \infty), \\
\mathrm{D}\left(\widetilde{T}_{\alpha}\right) & =\left\{\eta \in H^{4}((0, \infty)) \cap H_{0}^{1}((0, \infty)): f^{\prime \prime}(0)-\alpha f^{\prime}(0)=0\right\} .
\end{aligned}
$$

The boundary values at 0 are well defined due to the Sobolev embedding $H^{4}((0, \infty)) \hookrightarrow C^{3}([0, \infty))$. Considering the general solution (76) of the differential equation $f^{\prime \prime \prime \prime \prime}=k^{4} f$ and imposing the integrability requirement (i.e. $C_{1}, C_{4}=0$ ) together with the boundary conditions at 0 , we arrive at the condition

$$
k=\frac{1}{2^{1 / 2}} \alpha e^{i \pi / 4} .
$$

In order to make this equation compatible with (75), we see that the point spectrum of $T_{\alpha}$ is not empty if, and only if, $\arg \alpha \in(3 \pi / 4,5 \pi / 4)$, or equivalently, $\Re \alpha<-|\Im \alpha|$. If this condition is satisfied, $T_{\alpha}$ possesses one eigenvalue corresponding to the right-hand side put to the power 4 . Summing up,

$$
\sigma_{\mathrm{p}}\left(T_{\alpha}\right)= \begin{cases}\left\{-\frac{1}{4} \alpha^{4}\right\} & \text { if } \quad \Re \alpha<-|\Im \alpha| \\ \varnothing & \text { if } \quad \Re \alpha \geq-|\Im \alpha| .\end{cases}
$$


Notice that the arguments of $\alpha$ for which the point spectrum is empty correspond to real points intersecting the half-axis $[0,+\infty)=\sigma_{\text {ess }}\left(T_{\alpha}\right)$. So any eigenvalue of $T_{\alpha}$, if it exists, is necessarily discrete.

In particular, choosing $\alpha:=e^{i \theta}$ and varying $\theta \in(3 \pi / 4,5 \pi / 4)$, any boundary point of the closed disk $\left\{\lambda \in \mathbb{C}:|\lambda| \leq \frac{1}{4}\right\}$ except for the point $\lambda=\frac{1}{4}$ will be an eigenvalue of $H_{4 \pi \alpha \delta}$. This proves the optimality of Theorem 1 for $d=3$ because $\|4 \pi \alpha \delta\|_{L^{1}\left(\mathbb{R}^{3}\right)}=4 \pi|\alpha|=4 \pi$.

Remark 3. One can also justify the optimality of the constant in the eigenvalue bound corresponding to the inclusion (3) for $d=3$ in the limit $\varepsilon \rightarrow 0^{+}$by considering the following family of regular but singularly scaled potentials

$$
\delta_{\varepsilon}(x):= \begin{cases}\frac{1}{4 \pi \varepsilon^{3}} & \text { if }|x|<\varepsilon, \\ 0 & \text { otherwise }\end{cases}
$$

Of course $\delta_{\varepsilon} \rightarrow \delta$ in the sense of distributions as $\varepsilon \rightarrow 0^{+}$. What is more, restricting to radially symmetric eigenfunctions, the point spectrum of $H_{4 \pi \alpha \delta_{\varepsilon}}$ converges to the point spectrum of $H_{4 \pi \alpha \delta}$ as $\varepsilon \rightarrow 0^{+}$. This can be checked by explicitly solving the radial parts of the differential equations of the eigenvalue problem in the separate intervals where $\delta_{\varepsilon}$ is constant and matching the solutions at the interface point $r=\varepsilon$.

\section{References}

[1] A. A. Abramov, A. Aslanyan, and E. B. Davies, Bounds on complex eigenvalues and resonances, J. Phys. A: Math. Gen. 34 (2001), 57-72.

[2] M. S. Abramowitz and I. A. Stegun, eds., Handbook of mathematical functionsue, Dover, New York, 1965.

[3] R. A. Adams, Sobolev spaces, Academic Press, New York, 1975.

[4] J. Arazy and L. Zelenko, Virtual eigenvalues of the high order Schrödinger operator. I, Integ. Equ. Oper. Theory 55 (2006), no. 2, 189-231.

[5] _ Virtual eigenvalues of the high order Schrödinger operator. II, Integ. Equ. Oper. Theory 55 (2006), no. 3, 305-345.

[6] J. M. Arrieta, F. Ferraresso, and P. D. Lamberti, Spectral analysis of the biharmonic operator subject to Neumann boundary conditions on dumbbell domains, Integ. Equ. Oper. Theory $\mathbf{8 9}$ (2017), no. 3, 377-408.

[7] J.-C. Cuenin, Eigenvalue bounds for Dirac and fractional Schrödinger operators with complex potentials, J. Funct. Anal. 272 (2017), no. 7, 2987-3018.

[8] D. E. Edmunds and W. D. Evans, Spectral theory and differential operators, Oxford University Press, Oxford, 1987.

[9] T. Ekholm and A. Enblom, Critical Hardy-Lieb-Thirring inequalities for fourth-order operators in low dimensions, Lett. Math. Phys. 94 (2010), no. 3, 293-312.

[10] A. Enblom, Estimates for eigenvalues of Schrödinger operators with complex-valued potentials, Lett. Math. Phys. 106 (2016), no. 2, 197-220.

[11] W. D. Evans and R. T. Lewis, Counting eigenvalues of biharmonic operators with magnetic fields, Math. Nachr. 278 (2005), no. 12-13, 1524-1537.

[12] L. Fanelli and D. Krejčiřík, Location of eigenvalues of three-dimensional non-self-adjoint Dirac operators, Lett. Math. Phys. (2019). 
[13] L. Fanelli, D. Krejčiřík, and L. Vega, Spectral stability of Schrödinger operators with subordinated complex potentials, J. Spectr. Theory 8 (2018), 575-604.

[14] C. Förster and J. Östensson, Lieb-Thirring inequalities for higher order differential operators, Math. Nachr. 281 (2008), no. 2, 199-213.

[15] R. L. Frank, Eigenvalue bounds for Schrödinger operators with complex potentials, Bull. Lond. Math. Soc. 43 (2011), 745-750.

[16] I. S. Gradshteyn and I. M. Ryzhik, Table of integrals, series, and products, seventh ed., Elsevier/Academic Press, Amsterdam, 2007.

[17] A. Hulko, On the number of eigenvalues of the biharmonic operator on $\mathbb{R}^{3}$ perturbed by a complex potential, Rep. Math. Phys. 81 (2018), no. 3, 373-383.

[18] T. Kato, Wave operators and similarity for some non-selfadjoint operators, Math. Ann. 162 (1966), 258-279.

[19] E. H. Lieb and M. Loss, Analysis, American Mathematical Society, Providence, Rhode Island, 1997.

[20] Y. Netrusov and T. Weidl, On Lieb-Thirring inequalities for higher order operators with critical and subcritical powers, Comm. Math. Phys. 182 (1996), no. 2, 355-370.

[21] M. Reed and B. Simon, Methods of modern mathematical physics, II. Fourier analysis.Selfadjointness, Academic Press, New York, 1975.

[22] B. Simon, Quantum mechanics for Hamiltonians defined by quadratic forms, Princeton Univ. Press., New Jersey, 1971.

[23] T. Weidl, Remarks on virtual bound states for semi-bounded operators, Comm. Partial Differential Equations 24 (1999), no. 1-2, 25-60. 